\title{
An internally and externally validated nomogram for predicting the risk of irinotecan-induced severe neutropenia in advanced colorectal cancer patients
}

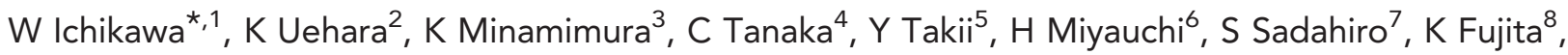 \\ T Moriwaki $^{9}$, M Nakamura ${ }^{10}$, T Takahashi $^{1}$, A Tsuji ${ }^{11}, \mathrm{~K}$ Shinozaki $^{12}$, S Morita ${ }^{13}$, Y Ando ${ }^{14}$, Y Okutani $^{15}$, \\ M Sugihara ${ }^{16}$, T Sugiyama $^{17}$, Y Ohashi ${ }^{18}$ and Y Sakata ${ }^{19}$
}

\begin{abstract}
${ }^{1}$ Division of Medical Oncology, Department of Medicine, Showa University, School of Medicine, 1-5-8 Hatanodai, Shinagawa-ku, Tokyo 142-8555, Japan; ${ }^{2}$ Division of Surgical Oncology, Department of Surgery, Nagoya University Graduate School of Medicine, 65 Tsurumai-cho, Showa-ku, Nagoya 466-8560, Japan; ${ }^{3}$ Department of Surgery, Mitsui Memorial Hospital, Kanda-Izumi-cho 1, Chiyoda-ku, Tokyo 101-8643, Japan; ${ }^{4}$ Department of Surgery, Gifu Prefectural General Medical Centre, 4-6-1 Noishiki, Gifu 500-8717, Japan; ${ }^{5}$ Department of Surgery, Niigata Cancer Centre Hospital, 2-15-3 Kawagishi-cho, Chuo-ku, Niigata 951-8566, Japan; ${ }^{6}$ Department of Frontier Surgery, Graduate School of Medicine, Chiba University, 1-8-1 Inohana, Chuo-ku, Chiba 2608670, Japan; ${ }^{7}$ Department of Surgery, Tokai University, 143 Shimoyasuya, Isehara 259-1193, Japan; ${ }^{8}$ Institute of Molecular Oncology, Showa University, 1 5-8 Hatanodai, Shinagawa-ku, Tokyo 142-8555, Japan; 'Division of Gastroenterology, University of Tsukuba, 1-1-1 Tennodai, Tsukuba 305-8575, Japan; ${ }^{10}$ Comprehensive Cancer Centre, Aizawa Hospital, 2-5-1 Honjo, Matsumoto 390-8510, Japan; ${ }^{11}$ Department of Medical Oncology, Kobe City Medical Centre General Hospital, 2-1-1 Minatojimaminamimachi, Chuo-ku, Kobe 650-0047, Japan; ${ }^{12}$ Division of Clinical Oncology, Hiroshima Prefectural Hospital, 1-5-54 Ujina-Kanda, Minami-ku, Hiroshima 734-8530, Japan; ${ }^{13}$ Department of Biomedical Statistics and Bioinformatics, Kyoto University Graduate School of Medicine, Yoshida-Konoe-cho, Sakyo-ku, Kyoto 606-8501, Japan; ${ }^{14}$ Department of Clinical Oncology and Chemotherapy, Nagoya University Hospital, 65 Tsurumai-cho, Showa-ku, Nagoya 466-8560, Japan; ${ }^{15}$ Medical Affairs Department, Daiichi Sankyo, 3-5-1 Nihonbashi-Honcho, Chuo-ku 103-8426, Tokyo, Japan; ${ }^{16}$ Clinical Data \& Biostatistics Department, Daiichi Sankyo, 1-2-58 Hiromachi, Shinagawa-ku, Tokyo 140-8710, Japan; ${ }^{17}$ Department of Obstetrics and Gynaecology, Iwate Medical University School of Medicine, 19-1 Uchimaru, Morioka 020-8505, Japan; ${ }^{18}$ Department of Integrated Science and Engineering for Sustainable Society, Faculty of Science and Engineering, Chuo University, 1-13-27 Kasuga, Tokyo 112-8551, Japan and ${ }^{19}$ CEO, Misawa City Hospital, 164-65, Aza Horiguchi, Oaza Misawa, Misawa, Aomori 033-0022, Japan
\end{abstract}

Background: In Asians, the risk of irinotecan-induced severe toxicities is related in part to UGT1A1*6 (UGT, UDP glucuronosyltransferase) and UGT1A1*28, variant alleles that reduce the elimination of SN-38, the active metabolite of irinotecan. We prospectively studied the relation between the UGT1A1 genotype and the safety of irinotecan-based regimens in Japanese patients with advanced colorectal cancer, and then constructed a nomogram for predicting the risk of severe neutropenia in the first treatment cycle.

Methods: Safety data were obtained from 1312 patients monitored during the first 3 cycles of irinotecan-based regimen in a prospective observational study. In development of the nomogram, multivariable logistic regression analysis was used to test the associations of candidate factors to severe neutropenia in the first cycle. The final nomogram based on the results of multivariable analysis was constructed and validated internally using a bootstrapping technique and externally in an independent data set $(n=350)$.

Results: The UGT1A1 genotype was confirmed to be associated with increased risks of irinotecan-induced grade 3 or 4 neutropenia and diarrhoea. The final nomogram included type of regimen, administered dose of irinotecan, gender, age, UGT1A1 genotype, Eastern Cooperative Oncology Group performance status, pre-treatment absolute neutrophil count, and total bilirubin level. The model was validated both internally (bootstrap-adjusted concordance index, 0.69) and externally (concordance index, 0.70).

Conclusions: Our nomogram can be used before treatment to accurately predict the probability of irinotecan-induced severe neutropenia in the first cycle of therapy. Additional studies should evaluate the effect of nomogram-guided dosing on efficacy in patients receiving irinotecan.

*Correspondence: Dr W Ichikawa; E-mail: wataru@med.showa-u.ac.jp

Received 5 December 2014; revised 16 February 2015; accepted 7 March 2015; published online 16 April 2015

(c) 2015 Cancer Research UK. All rights reserved 0007-0920/15

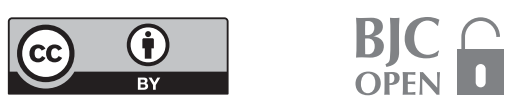


Irinotecan is an effective drug for advanced colorectal cancer, as a single agent (Shimada et al, 1993) or in combination with a fluoropyrimidine (Goto et al, 2006; Muro et al, 2010; Komatsu et al, 2011), with or without a monoclonal antibody (Saltz, 2013). Irinotecan is a pro-drug, and its active metabolite SN-38 has both antitumour activities and toxicities (Senter et al, 2001; Xu et al, 2002). SN-38 is inactivated into SN-38 glucuronide (SN-38G) mainly by UDP-glucuronosyltransferase 1A1 (UGT1A1) (Iyer et al, 1998).

Genetic polymorphisms in $U G T 1 A 1$, such as $U G T 1 A 1^{\star} 28$ in Caucasians and Asians and $U G T 1 A 1^{\star} 6$ only in Asians, contribute to interpatient variability in the pharmacokinetics and toxicities of irinotecan, particularly severe neutropenia (Ando et al, 2000; Innocenti et al, 2004; Minami et al, 2007; Innocenti et al, 2009; Chen et al, 2014). In 2005, the US Food and Drug Administration recommended that the package insert of irinotecan be amended to encourage the use of a reduced starting dose in patients homozygous for $U G T 1 A 1^{\star} 28\left({ }^{\star} 28{ }^{*} 28\right)$. In 2008 , the Ministry of Health, Labour, and Welfare of Japan likewise recommended that the package insert be revised to warn of the risk of severe irinotecan-related neutropenia in Japanese patients who are either homozygous for $U G T 1 A 1^{\star} 6$ or $U G T 1 A 1^{*} 28$ or heterozygous for both $U G T 1 A 1^{\star} 6$ and $U G T 1 A 1^{\star} 28$. Subsequently, diagnostic genotyping for the $U G T 1 A 1^{\star} 6$ and $U G T 1 A 1^{*} 28$ was approved in Japan and covered by health insurance.

However, factors other than the UGT1A1 genotype may contribute to irinotecan-induced severe toxicity such as neutropenia and diarrhoea. Other non-genetic factors, such as organ functions, age, gender, co-morbidities, and performance status (PS), should therefore be comprehensively considered in predicting the risk of severe irinotecan-related toxicities (Innocenti et al, 2004; Marcuello et al, 2004; Rouits et al, 2004; Innocenti and Ratain, 2006; Kweekel et al, 2008; Liu et al, 2008).

We designed a prospective observational study to evaluate the effects of UGT1A1 genotypes and non-genetic factors on the efficacy and safety of irinotecan-based regimens in Japanese patients with advanced colorectal cancer. Our primary objective was to demonstrate non-inferiority of the response to irinotecanbased regimens in terms of progression-free survival between patients harbouring $U G T 1 A 1^{\star} 6$ or $U G T 1 A 1^{\star} 28$ and patients without these polymorphisms. The secondary objective was to evaluate the relation between UGT1A1 genotype and the safety of irinotecan-based regimens. The results of the final analysis of outcomes will be available in 2015; however, we now report the results of a planned interim analysis of safety data from 1312 patients and describe the development of a nomogram for predicting the risk of irinotecan-induced severe neutropenia, with external validation using an independent cohort of 350 patients.

\section{MATERIALS AND METHODS}

Study design and patient eligibility. This multicentre, open-label, prospective, non-interventional, observational study was conducted at 299 sites in Japan. Eligible patients had to (1) have a histologically confirmed diagnosis of adenocarcinoma of the colon or rectum, (2) have advanced or metastatic disease that was not amenable to curative resection, (3) undergo genotyping for $U G T 1 A 1^{\star} 1,{ }^{\star} 6$ and ${ }^{\star} 28$ before treatment, and (4) be scheduled to receive FOLFIRI (Muro et al, 2010) (folinic acid $\left(200 \mathrm{mg} \mathrm{m}^{-2}\right.$ ) and irinotecan $\left(150 \mathrm{mg} \mathrm{m}^{-2}\right)$ and then a bolus injection of 5fluorouracil $\left(400 \mathrm{mg} \mathrm{m}^{-2}\right.$ ) on day 1 and a continuous infusion of 5-fluorouracil $\left(2400 \mathrm{mg} \mathrm{m}^{-2}\right)$ over $46 \mathrm{~h}$, repeated every 2 weeks), IRIS (Muro et al, 2010; Komatsu et al, 2011) (irinotecan $\left(125 \mathrm{mg} \mathrm{m}^{-2}\right)$ on days 1 and 15 and S-1 (40-60 mg according to body surface area) twice daily for 2 weeks, repeated every 4 weeks), SIR (Goto et al, 2006) (irinotecan $\left(150 \mathrm{mg} \mathrm{m}^{-2}\right.$ ) on day 1 and S-1 twice daily for 2 weeks, repeated every 3 weeks), or bi-weekly irinotecan monotherapy $\left(150 \mathrm{mg} \mathrm{m}^{-2}\right)$, with or without molecular targeted agents and irrespective of the treatment line. The recommended dose of irinotecan for FOLFIRI is $150 \mathrm{mg} \mathrm{m}^{-2}$ for Japanese, which is less than the $180 \mathrm{mg} \mathrm{m}^{-2}$ dose recommended for Caucasians, and the recommended treatment schedule in Japan ( $150 \mathrm{mg} \mathrm{m}^{-2}$, on day 1 , repeated every 2 weeks) differs from that in Europe and the US $\left(350 \mathrm{mg} \mathrm{m}^{-2}\right.$, on day 1 , repeated every 3 weeks). Eligible patients also had to have adequate bone marrow reserve and liver and renal functions and provide written informed consent before enrolment. Patients were excluded if they had conditions precluding the use of irinotecan-based regimens, an Eastern Cooperative Oncology Group (ECOG) PS of 3 or 4 , or a history of pelvic irradiation. The protocol was reviewed and approved by an independent ethics committee or the institutional review board of each participating centre. The study was conducted in accordance with the Declaration of Helsinki and local ethical and legal requirements. This observational study was registered with the website http:// www.ClinicalTrials.gov (reference identification: NCT 01039506) and the website http://www.clinicaltrials.jp/ (reference identification: JapicCTI-090945).

Treatment and evaluation of safety. The choice of treatment regimen and dosage was left to the investigator's discretion. As described previously, 4 weeks of treatment was considered 1 cycle for FOLFIRI (Muro et al, 2010), IRIS (Muro et al, 2010; Komatsu et al, 2011), or irinotecan monotherapy (Shimada et al, 1993), and 3 weeks of treatment was considered 1 cycle for SIR (Goto et al, 2006). Relative dose-intensity was defined as the ratio of the cumulative administered dose to the scheduled total dose for the first 3 cycles of treatment (Shimada et al, 1993; Goto et al, 2006; Muro et al, 2010; Komatsu et al, 2011). The UGT1A1 genetic profiles were categorised into three groups as described previously (Sato et al, 2011): wild-type $\left({ }^{*} 1 /{ }^{*} 1\right)$, heterozygous $\left({ }^{*} 1 /{ }^{*} 6,{ }^{*} 1 /{ }^{*} 28\right.$ ), and homozygous $\left({ }^{*} 6 /{ }^{*} 6,{ }^{*} 6 /{ }^{*} 28,{ }^{*} 28 /{ }^{*} 28\right)$. Clinical findings and laboratory tests were evaluated every 2 weeks during the first 3 cycles. (Evaluations at the times of drug administration were mandatory.) After the first 3 cycles, evaluations for safety were performed according to standard clinical practice. Baseline data at registration and detailed toxicities during the first 3 cycles were prospectively recorded via a web-based electronic data collection system. The types and severities of adverse events were graded according to the National Cancer Institute's Common Terminology Criteria for Adverse Events (NCI-CTCAE), version 3.0, and the highest grade of each adverse event was recorded. Safety analyses focused on neutropenia and diarrhoea because these are the most common dose-limiting toxicities associated with irinotecan-based regimens (Ando et al, 2000; Innocenti et al, 2004; Kweekel et al, 2008).

Statistical analysis. The relative risk (RR) of grade 3 or 4 neutropenia or diarrhoea in the heterozygous or homozygous groups was calculated as compared with that in the wild-type group. Fisher's exact test was used for group comparisons.

Nomogram model building and validation. Multivariable logistic regression analysis was performed to examine the relations of various factors to the occurrence of severe neutropenia of grade 3 or 4 in the first cycle. The multivariable logistic regression model included the following factors: treatment line (first, second, or later line), regimen (FOLFIRI, irinotecan $+\mathrm{S}-1$, or irinotecan monotherapy), administered dose of irinotecan, gender, age, UGT1A1 genotype (wild-type, heterozygous, or homozygous), ECOG PS (0, 1 , or 2), molecular targeted agents, prior surgery, prior radiation, pre-treatment absolute neutrophil count, and pre-treatment total bilirubin level, based on previous reports (Innocenti et al, 2004; Marcuello et al, 2004; Rouits et al, 2004; Innocenti and Ratain, 
2006; Hoskins et al, 2007; Kweekel et al, 2008; Liu et al, 2008; McLeod et al, 2010; Shiozawa et al, 2013). Missing values for the pre-treatment absolute neutrophil count $(n=68,5.2 \%)$ and total bilirubin levels ( $n=127,9.7 \%)$ were imputed by median imputation (Little and Rubin, 2002) for each UGT1A1 genotype. All categorical predictors were modelled using dummy variables, while all continuous predictors were modelled using restricted quadratic splines (Greenland, 1995) based on 2 knots for the tertiles to relax linearity assumptions. The final model was chosen on the basis of variables that had $P<0.10$ on a backward step-down selection process.

Nomogram validation consisted of discrimination and calibration. Discrimination refers to a nomogram model's ability to correctly distinguish two classes of outcomes. First, for internal validation, we used both a bootstrap method with 1000 resamples and a 10 -fold cross-validation with 200 repetitions to estimate the bias-corrected or over-fitting corrected predictive accuracy of the model, which is expressed as the concordance index (c-index). Second, we assessed calibration, which compares the predicted probability with the observed outcome in 10 groups partitioned by the decile of the predicted probabilities.

External validation was performed by applying the prediction model to an independent cohort of 350 patients with advanced colorectal cancer who met the same eligibility criteria as the original cohort and were from six independent sites in Japan. The protocol for external validation was also reviewed and approved in each institution. Discriminative power and calibration in the independent cohort were also evaluated.

SAS version 9.2 (SAS Institute, Cary, NC, USA) was used to perform all statistical analyses. All $P$ values were two-sided, and $P$ values of $<0.05$ were considered to indicate statistical significance.

\section{RESULTS}

Patient and treatment characteristics. Between October 2009 and March 2012, a total of 1376 patients were enrolled. Sixty-four patients were excluded for the following reasons: 42 had no case report forms submitted by the investigators; 15 did not receive irinotecan-based regimens; 4 patients did not meet the inclusion criteria; and 3 withdrew consent after registration. Data from the remaining 1312 patients were included in safety analysis and nomogram development. The baseline characteristics are summarised in Table 1. The UGT1A1 genotype was wild-type in $47.9 \%$ of the patients, heterozygous in $41.1 \%$, and homozygous in $11.1 \%$. Nearly $80 \%$ of the patients received irinotecan-based regimens as second- or later-line chemotherapy. The rate of received regimen type was similar among the three groups according to the UGT1A1 genotype. Similar to a previous study (Sai et al, 2004; Liu et al, 2008), the median pre-treatment total bilirubin level was higher in the homozygous group $\left(0.80 \mathrm{mg} \mathrm{dl}^{-1}\right)$ than in the wild-type and heterozygous groups (both $0.60 \mathrm{mg} \mathrm{dl}^{-1}$ ).

The median administered dose of irinotecan in the first cycle of FOLFIRI was approximately $150 \mathrm{mg} \mathrm{m}^{-2}$ in the wild-type and heterozygous groups, as compared with $122.5 \mathrm{mg} \mathrm{m}^{-2}$ in the homozygous group, which is nearly $20 \%$ less than the recommended dose for FOLFIRI in Japan $\left(150 \mathrm{mg} \mathrm{m}^{-2}\right.$ ) (Muro et al, 2010) (Supplementary Table S1). In patients given FOLFIRI, the 3 -cycle relative dose-intensities of irinotecan in the homozygous group (55.9\%) were lower than those in the wild-type (66.3\%) and heterozygous groups (64.1\%), irrespective of regimen.

Safety. The incidences of grade 3 or 4 neutropenia in the first cycle and entire treatment cycle (up to the third cycle) were, respectively, $18.9 \%$ and $25.2 \%$ in the wild-type group, $26.5 \%$ and $34.1 \%$ in the heterozygous group, and $42.1 \%$ and $49.0 \%$ in the homozygous group (Figure 1). Severe neutropenia of grade 3 or 4 was more common in the homozygous group (RR, 2.220; $P<0.0001$ in the first cycle; RR, 1.946; $P<0.0001$ in the entire cycle) and heterozygous group (RR, 1.400; $P=0.0024$ in the first cycle; RR, $1.357 ; P=0.0010$ in the entire cycle) than in the wildtype group.

Febrile neutropenia developed in 30 (2.3\%) of the 1312 eligible patients (11 patients in the wild-type group, 9 in the heterozygous group, and 10 in the homozygous group).

The incidences of grade 3 or 4 diarrhoea in the entire cycle were $4.0 \%, 3.3 \%$, and $7.6 \%$ in the wild-type, heterozygous, and homozygous group, respectively (Figure 2). The incidence of severe diarrhoea was significantly higher in the homozygous group (5.5\%) than in the wild-type group $(2.1 \%)$ only in the first cycle (RR, 2.665; $P=0.0405$ ).

The incidences of severe toxicities in the homozygous group decreased in parallel to the number of treatment cycles (incidences of neutropenia and diarrhoea: $42.1 \%$ and $5.5 \%$ in first cycle, $23.9 \%$ and $1.8 \%$ in second cycle, and $14.4 \%$ and $2.1 \%$ in third cycle, respectively).

Nomogram development based on the final prediction model and validation. The results of multivariable logistic regression analysis of factors potentially related to severe neutropenia in first cycle of irinotecan-based regimens are shown in Supplementary Table S2. After backward step-down variable selection, treatment line, molecular targeted agents, prior surgery, and prior radiation were excluded from the final prediction model.

The final prediction model for severe neutropenia in the first cycle of irinotecan-based regimens is shown in Table 2. Significant factors $(P<0.10)$ included regimen (FOLFIRI $v s$ irinotecan $+\mathrm{S}-1$ $v s$ irinotecan monotherapy), administered dose of irinotecan, gender (male $v s$ female), age, UGT1A1 genotype (wild-type vs heterozygous $v s$ homozygous), ECOG PS ( 0 vs 1 vs $\geq 2)$, pretreatment absolute neutrophil count, and pre-treatment total bilirubin level.

The bootstrap-corrected c-index and the c-index after 10-fold cross-validation were 0.693 and 0.668 , respectively. The nomogram for predicting the probability of severe neutropenia during the first cycle of irinotecan-based regimens was internally validated as shown in Figure 3. The bootstrap-corrected calibration slope in the internal cohort is shown in Figure 4, which indicated good agreement between the predicted and observed probabilities of severe neutropenia.

Using data from an independent cohort set $(n=350)$, we attempted to validate the nomogram externally. The incidence of severe neutropenia was $25.7 \%$ in the external validation cohort. Patients' characteristics were similar in the internal and external validation cohorts, with the exceptions of PS and regimens (Supplementary Table S3). The nomogram also demonstrated good accuracy for predicting the risk of severe neutropenia in the external validation cohort, with a c-index of 0.702 . The calibration slope in the external validation cohort was 1.1907 , and the intercept -0.0295 .

\section{DISCUSSION}

We studied the relation between the safety of irinotecan-based regimens and UGT1A1 genotype and developed a nomogram to predict the risk of irinotecan-induced severe neutropenia in 1312 advanced colorectal cancer patients registered in a prospective observational study. To our knowledge, our study is the largest prospective study of its type to be performed in Asia; moreover, this is the first nomogram to be validated internally and externally.

Our pre-planned interim analysis of safety confirmed that the UGT1A1 homozygous and heterozygous genotypes were associated with a higher risk of severe neutropenia than the UGT1A1 wild- 
Table 1. Baseline characteristics

\section{UGT1A1 genotype group}

\begin{tabular}{|c|c|c|c|c|}
\hline Characteristics & Wild-type $(N=628)$ & Heterozygous $(N=539)$ & Homozygous $(N=145)$ & All $(N=1312)$ \\
\hline Median age, years (IQR) & $67(61,73)$ & $66(60,73)$ & $68(61,74)$ & $67(61,73)$ \\
\hline \multicolumn{5}{|l|}{ Gender, $n(\%)$} \\
\hline Male & $371(59.1)$ & $362(67.2)$ & 85 (58.6) & $818(62.3)$ \\
\hline Female & $257(40.9)$ & $177(32.8)$ & $60(41.4)$ & $494(37.7)$ \\
\hline Median BSA, $\mathrm{m}^{2}$ (IOR) & $1.52(1.40,1.66)$ & $1.57(1.44,1.68)$ & $1.54(1.39,1.67)$ & $1.54(1.42,1.67)$ \\
\hline \multicolumn{5}{|l|}{ UGT1A1 genetic profile, n (\%) } \\
\hline \multicolumn{5}{|l|}{ Wild-type group } \\
\hline$* 1 / * 1$ & $628(100.0)$ & & & $628(47.9)$ \\
\hline Heterozygous group & & $539(100.0)$ & & $539(41.1)$ \\
\hline $\begin{array}{l}* 1 / * 6 \\
* 1 / * 28\end{array}$ & & $\begin{array}{l}308(57.1) \\
231(42.9)\end{array}$ & & $\begin{array}{l}308(23.5) \\
231(17.6)\end{array}$ \\
\hline Homozygous group & & & $145(100.0)$ & $145(11.1)$ \\
\hline $\begin{array}{l}* 6 / * 6 \\
* 28 / * 28 \\
* 6 / * 28\end{array}$ & & & $\begin{array}{l}50(34.5) \\
23(15.9) \\
72(49.7)\end{array}$ & $\begin{array}{l}50(3.8) \\
23(1.8) \\
72(5.5)\end{array}$ \\
\hline \multicolumn{5}{|l|}{ ECOG PS, $n(\%)$} \\
\hline 0 & $457(72.8)$ & 401 (74.4) & $109(75.2)$ & 967 (73.7) \\
\hline 1 & $142(22.6)$ & $118(21.9)$ & $29(20.0)$ & $289(22.0)$ \\
\hline 2 & $29(4.6)$ & $20(3.7)$ & $7(4.8)$ & $56(4.3)$ \\
\hline \multicolumn{5}{|l|}{ Treatment line, $n(\%)$} \\
\hline First & $138(22.0)$ & $125(23.2)$ & $21(14.5)$ & $284(21.6)$ \\
\hline Second or later & $490(78.0)$ & $414(76.8)$ & $124(85.5)$ & $1028(78.4)$ \\
\hline \multicolumn{5}{|l|}{ Regimen, $n(\%)$} \\
\hline FOLFIRI & $395(62.9)$ & $349(64.7)$ & $96(66.2)$ & $840(64.0)$ \\
\hline Irinotecan + S-1 & $160(25.5)$ & $132(24.5)$ & $32(22.1)$ & $324(24.7)$ \\
\hline Irinotecan monotherapy & $73(11.6)$ & $58(10.8)$ & $17(11.7)$ & $148(11.3)$ \\
\hline \multicolumn{5}{|l|}{ Molecular targeted agents, $n(\%)$} \\
\hline None & $236(37.6)$ & $209(38.8)$ & $51(35.2)$ & $496(37.8)$ \\
\hline Anti-VEGF mAb & $274(43.6)$ & $223(41.4)$ & $64(44.1)$ & $561(42.8)$ \\
\hline Anti-EGFR mAb & $118(18.8)$ & 107 (19.9) & $30(20.7)$ & $255(19.4)$ \\
\hline \multicolumn{5}{|l|}{ Prior therapy, $n(\%)$} \\
\hline Surgery & $526(83.8)$ & $456(84.6)$ & $116(80.0)$ & 1098 (83.7) \\
\hline Radiation & $46(7.3)$ & $38(7.1)$ & 15 (10.3) & $99(7.5)$ \\
\hline Chemotherapy & $481(76.6)$ & $417(77.4)$ & $121(83.4)$ & $1019(77.7)$ \\
\hline Median WBC, $10^{2} \mathrm{~mm}^{-3}$ (IQR) & $53.5(42.6,66.8)$ & $53.5(42.0,69.0)$ & $52.0(41.3,63.5)$ & $53.0(42.0,67.0)$ \\
\hline Median ANC, $10^{2} \mathrm{~mm}^{-3}$ (IQR) & $31.3(23.0,41.7)$ & $31.6(23.3,42.5)$ & $31.0(24.0,41.0)$ & $31.3(23.1,42.0)$ \\
\hline Median platelet, $10^{4} \mathrm{~mm}^{-3}$ (IQR) & $18.7(14.3,24.4)$ & $19.1(14.6,24.0)$ & $18.3(14.5,23.5)$ & $18.8(14.5,24.1)$ \\
\hline Median total bilirubin level, $\mathrm{mg} \mathrm{dl}^{-1}$ (IQR) & $0.60(0.40,0.77)$ & $0.60(0.50,0.87)$ & $0.80(0.60,1.20)$ & $0.60(0.46 .0 .80)$ \\
\hline
\end{tabular}

Abbreviations: ANC = absolute neutrophil count; BSA = body surface area; ECOG PS=Eastern Cooperative Oncology Group performance status; EGFR=epidermal growth factor receptor; FOLFIRI = folinic acid, fluorouracil, and irinotecan; $I Q R=$ interquartile range; $\mathrm{mAb}=$ monoclonal antibody; UGT1A1=uridine diphosphate glucuronosyltransferase $1 \mathrm{~A} 1 ; \mathrm{VEGF}=$ vascular endothelial growth factor; $\mathrm{WBC}=$ white blood cells.

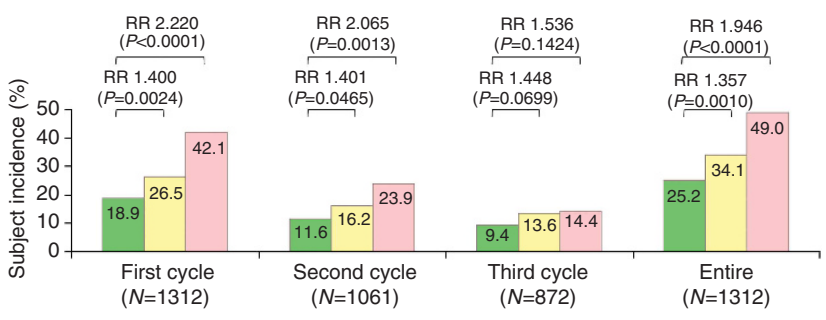

Figure 1. Subject incidences of grade 3 or 4 neutropenia according to UGT1A1 genotype. Green, yellow, and pink bars represent the incidences for patients harbouring UGT1A1 wild-type $\left({ }^{*} 1 /{ }^{*} 1\right)$, heterozygous $\left({ }^{*} 1 /{ }^{*} 6,{ }^{*} 1 /{ }^{*} 28\right)$, and homozygous $\left({ }^{*} 6 /{ }^{*} 6,{ }^{*} 6 /{ }^{*} 28, * 28 /{ }^{*} 28\right)$ genotypes, respectively. Abbreviation: $R R=$ relative risk.

type genotype, consistent with the results of many previous studies (Ando et al, 2000; Innocenti et al, 2004; Marcuello et al, 2004; Rouits et al, 2004; Innocenti and Ratain, 2006; Hoskins et al, 2007;

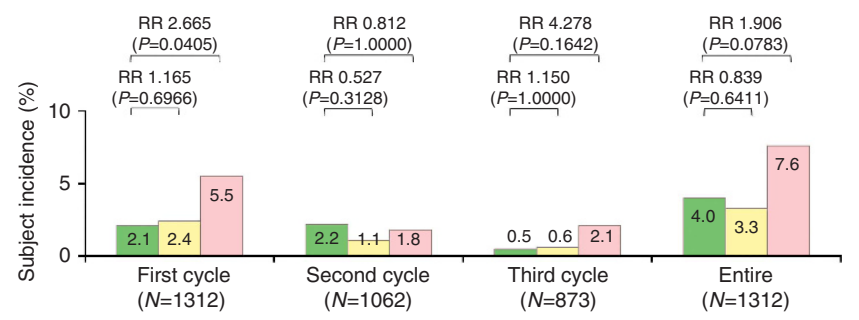

Figure 2. Subject incidences of grade 3 or 4 diarrhoea according to UGT1A1 genotype. Green, yellow, and pink bars represent the incidences for patients harbouring UGT1A1 wild-type $\left({ }^{*} 1 /{ }^{*} 1\right)$, heterozygous $\left({ }^{*} 1 /{ }^{*} 6,{ }^{*} 1 /{ }^{*} 28\right)$, and homozygous $\left({ }^{*} 6 /{ }^{*} 6,{ }^{*} 6 /{ }^{*} 28,{ }^{*} 28 /{ }^{*} 28\right)$ genotypes, respectively. Abbreviation: RR $=$ relative risk.

Minami et al, 2007; Kweekel et al, 2008; Liu et al, 2008; Innocenti et al, 2009; McLeod et al, 2010; Chen et al, 2014). In safety results, regardless of the UGT1A1 genotype, the subject incidence of severe 
Table 2. Final prediction model based on multivariable logistic regression analysis for severe neutropenia in the first cycle $(N=1312)$

\begin{tabular}{|c|c|c|c|c|c|c|c|}
\hline & \multicolumn{7}{|c|}{ Odds ratio } \\
\hline & $N$ & $n(\%)$ & Estimate & $(95 \% \mathrm{Cl})$ & $P$ value & Overall $P$ & c-index ${ }^{a}$ \\
\hline Regimen & & & & & & & $0.693(0.668$ \\
\hline FOLFIRI & 840 & $241(28.7)$ & 1 & - & & 0.0015 & \\
\hline Irinotecan + S-1 & 324 & $53(16.4)$ & 0.546 & $(0.375,0.794)$ & 0.0015 & & \\
\hline Irinotecan monotherapy & 148 & $29(19.6)$ & 0.579 & $(0.367,0.914)$ & 0.0190 & & \\
\hline Administered irinotecan dose $\left(\mathrm{mg} \mathrm{m}^{-2}\right)^{\mathbf{b}}$ & 1312 & $323(24.6)$ & - & - & - & 0.0024 & \\
\hline \multicolumn{8}{|l|}{ Gender } \\
\hline $\begin{array}{l}\text { Male } \\
\text { Female }\end{array}$ & $\begin{array}{l}818 \\
494\end{array}$ & $\begin{array}{l}184(22.5) \\
139(28.1)\end{array}$ & $\begin{array}{l}0.686 \\
1\end{array}$ & $\begin{array}{c}(0.521,0.902) \\
-\end{array}$ & $\begin{array}{c}0.0070 \\
-\end{array}$ & 0.0070 & \\
\hline Age (years) ${ }^{b}$ & 1312 & $323(24.6)$ & - & - & - & 0.0478 & \\
\hline \multicolumn{8}{|l|}{ UGT1A1 genotype } \\
\hline $\begin{array}{l}\text { Wild-type } \\
\text { Heterozygous } \\
\text { Homozygous }\end{array}$ & $\begin{array}{l}628 \\
539 \\
145\end{array}$ & $\begin{array}{r}119(18.9) \\
143(26.5) \\
61(42.1)\end{array}$ & $\begin{array}{l}1 \\
1.624 \\
3.343\end{array}$ & $\begin{array}{c}- \\
(1.217,2.167) \\
(2.191,5.100)\end{array}$ & $\begin{array}{r}-\overline{0} \\
0.0010 \\
<0.0001\end{array}$ & $<0.0001$ & \\
\hline \multicolumn{8}{|l|}{ ECOG PS } \\
\hline $\begin{array}{l}0 \\
1 \\
2\end{array}$ & $\begin{array}{r}967 \\
289 \\
56\end{array}$ & $\begin{array}{r}228(23.6) \\
80(27.7) \\
15(26.8)\end{array}$ & $\begin{array}{l}1 \\
1.330 \\
1.749\end{array}$ & $\begin{array}{c}-\overline{-} \\
(0.968,1.828) \\
(0.893,3.429)\end{array}$ & $\begin{array}{l}-\overline{0} \\
0.10387\end{array}$ & 0.0811 & \\
\hline Pre-treatment ANC $\left(\mathrm{mm}^{-3}\right)^{\mathbf{b}}$ & 1312 & $323(24.6)$ & - & - & - & 0.0005 & \\
\hline Pre-treatment total bilirubin level, $\left(\mathrm{mg} \mathrm{dl}^{-1}\right)^{\mathbf{b}}$ & 1312 & $323(24.6)$ & - & - & - & 0.0003 & \\
\hline \multicolumn{8}{|c|}{$\begin{array}{l}\text { Abbreviations: ANC }=\text { absolute neutrophil count; c-index }=\text { concordance index; } 95 \% \mathrm{Cl}=95 \% \text { confidence interval; ECOG PS = Eastern Cooperative Oncology Group performance status } \\
\text { FOLFIRI = folinic acid, fluorouracil, and irinotecan; UGT1A1 = uridine diphosphate glucuronosyltransferase } 1 \mathrm{~A} 1 . \\
\text { a Bootstrap-corrected c-index (c-index from } 10 \text {-fold cross-validation). } \\
\text { b }\end{array}$} \\
\hline
\end{tabular}

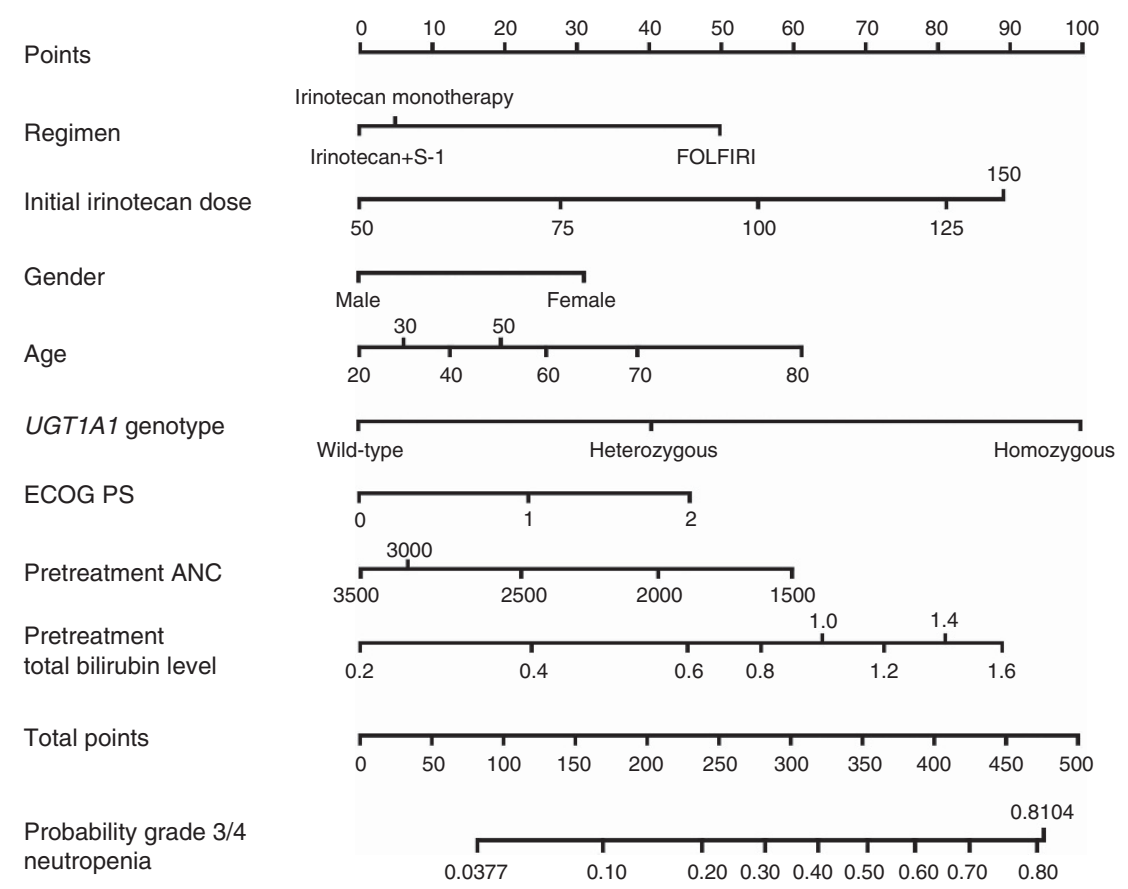

Figure 3. Nomogram for predicting the probability of irinotecan-induced severe neutropenia in the first cycle. To calculate the probability of severe (grade 3 or 4) neutropenia, first determine the value for each factor by drawing a vertical line from that factor to the point scale. Then, sum all individual values and draw a vertical line from the total point scale to the probability of severe neutropenia.

neutropenia successively decreased in the second and third treatment cycles (Figure 1). This decreasing incidence might have resulted from dose or schedule modifications at the physician's discretion, mainly based on the severity of neutropenia in the previous cycle. Moreover, the homozygous genotype was associated with a two-fold (RR, 2.220) higher risk of severe neutropenia in the first course as compared with wild-type, despite using a lower starting dose in the homozygous group. This finding suggests that 


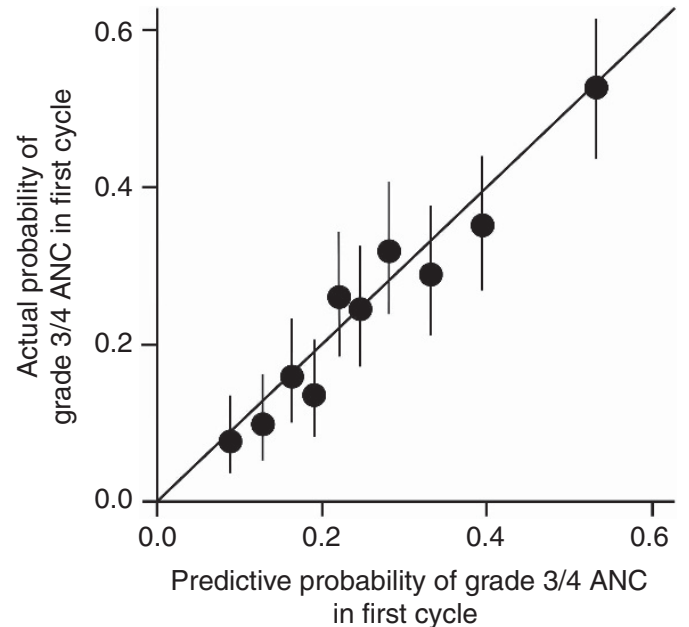

Figure 4. Nomogram model calibration curve for the internal validation cohort $(n=1312)$. Black line represents ideal fit, where the nomogram-predicted probability ( $x$-axis) matches the observed probability (y-axis). Closed circles and vertical bars represent the observed probabilities and $95 \% \mathrm{Cl}$ for 10 groups partitioned by the decile of the predicted probabilities. The bootstrap-corrected calibration slope of the regression line in the internal cohort is 1.0026 $(95 \% \mathrm{Cl} ; 0.8053,1.2038)$, with an intercept of $-0.0005(95 \% \mathrm{Cl}$; $-0.0485,0.0428)$. Abbreviation: $\mathrm{Cl}=$ confidence interval.

the risk of severe neutropenia induced by irinotecan-based regimens cannot be predicted solely on the basis of UGT1A1 genotype and the administered dose of irinotecan; potential effects of other non-genetic factors such as patients' clinical characteristics must also be considered.

The resulting nomogram demonstrated good accuracy for predicting the probability of severe neutropenia in the first cycle, with a bootstrap-corrected c-index of 0.693 and 0.702 for the internal and external validation cohorts, respectively. The c-index of 0.70 in the external validation cohort indicates that the accuracy of the nomogram for predicting severe neutropenia is $70 \%$, which is considered a clinically meaningful value. Additionally, the UGT1A1 genotype was the strongest predictor of severe neutropenia among factors included in the final prediction model. In the internal cohort, when only UGT1A1 genotype was used, the bootstrap-corrected c-index was 0.593 in the prediction model for severe neutropenia in the first cycle of irinotecan-based regimens (odds ratio, 1.545; $P=0.0020$ for heterozygous $v s$ wild-type; odds ratio, 3.106; $P<0.0001$ for homozygous $v s$ wild-type) (data not shown). The unacceptable discriminative power of the prediction model including only the UGT1A1 genotype suggests that our comprehensive approach incorporating non-genetic factors provides a more accurate prediction of the risk of severe neutropenia.

A prediction model for severe neutropenia was constructed only for the first cycle, using eight factors chosen by the multivariable logistic regression model (Table 2). We could not construct a prediction model for severe diarrhoea or febrile neutropenia owing to the limited number of patients with such toxicity. Although the total bilirubin level at the start of the first cycle was associated with the UGT1A1 genotype, both factors were independent predictors. In agreement with our findings, Innocenti et al (2009) reported that both the bilirubin level and UGT1A1 genotype were significant factors in a model including pre-treatment data to predict the risk of severe neutropenia, suggesting that each added predictive value.

Clinically, this internally and externally validated nomogram will most likely be useful for predicting the probability of irinotecan-induced severe neutropenia in patients with colorectal cancer. If the probability of severe neutropenia exceeds the clinically permissible range (e.g., $\geq 70 \%$ ), the starting dose of irinotecan should be reduced. The UGT1A1 genotype-directed dosing of irinotecan has been evaluated in patients receiving irinotecan-based therapy (Toffoli et al, 2010; Marcuello et al, 2011) or irinotecan monotherapy (Sato et al, 2011; Innocenti et al, 2014). Patients with a low probability of severe neutropenia (e.g., <30\%) as calculated with the nomogram using the standard dose of irinotecan can tolerate substantially higher doses. The concept of nomogram-based dosing of irinotecan should be explored in future clinical trials.

The association between the UGT1A1 genotype and severe diarrhoea remains controversial (Kweekel et al, 2008). Most of the previous studies retrospectively evaluated relatively small numbers of patients with different types of cancer who received various irinotecan-based regimens, although the $U G T 1 A 1^{*} 28 /{ }^{*} 28$ genotype was associated with a two-fold higher risk of diarrhoea than wildtype genotype in a meta-analysis limited to Caucasians with colorectal cancer (Liu et al, 2014). In our study, severe diarrhoea was significantly more common in the homozygous group than in the wild-type group only in the first cycle (RR, 2.665) (Figure 2). The significant association between severe diarrhoea and the UGT1A1 genotype in our study is attributed to focusing on 1312 patients with advanced colorectal cancer who received three irinotecan-based regimens, supporting that the UGT1A1 genotype may serve as a predictive marker for irinotecan-induced severe diarrhoea.

Our study had several limitations. First, our results are applicable to only Asians, because the recommended doses and schedules of irinotecan-based regimens differ between Japan and Western countries, $S-1$ is frequently used in Asia, and the $U G T 1 A 1^{*} 6$ allele is not found in Caucasians. Second, polymorphisms other than $U G T 1 A 1^{\star} 6$ or $U G T 1 A 1^{\star} 28$, such as UGT1A7, UGT1A9 (Carlini et al, 2005; Han et al, 2006; Hazama et al, 2013), $A B C B 1, A B C C 2, A B C G 2$, and SLCO1B1, have been suggested to be associated with toxicities induced by irinotecan-based regimens (Innocenti et al, 2009; Sai et al, 2010). Third, our nomogram can only be used to estimate the probability of irinotecan-induced severe neutropenia, but not efficacy. Although several metaanalyses have examined the correlation between UGT1A1 genotype and the efficacy of irinotecan-based regimens, including tumour response and survival (Palomaki et al, 2009; Dias et al, 2012; Liu et al, 2013; Dias et al, 2014), their results remain controversial. The results of our final analysis of outcomes, scheduled to be available in 2015, are expected to shed light on these and other unresolved issues.

In conclusion, our study provides pivotal evidence supporting the association between the UGT1A1 genotype and an increased risk of irinotecan-induced severe neutropenia and diarrhoea in Japanese patients with colorectal cancer. We developed and validated a clinically useful nomogram including UGT1A1 genotype and other non-genetic factors for predicting the risk of severe neutropenia in the first cycle of irinotecan-based chemotherapy. We believe that our study represents a great step toward the goal of precision medicine based on irinotecan pharmacogenetics.

\section{ACKNOWLEDGEMENTS}

We thank the patients and their families, and the investigators from the 299 participating institutions for their cooperation. We thank Koji Hirata for editorial assistance and Yu Sunakawa for helpful discussions. This study was funded by Daiichi Sankyo Co., Ltd., Tokyo, Japan. 


\section{CONFLICT OF INTEREST}

WI is a consultant/advisory board member of Daiichi Sankyo and Merck-Serono. KU, KM, YT, HM, SS, MN, AT, and KS report receiving commercial research funds from Daiichi Sankyo. TM reports receiving commercial research funds from Daiichi Sankyo, Taiho Pharmaceutical, and Sanofi. SM is a consultant/advisory board member of Daiichi Sankyo and Taiho Pharmaceutical, and reports receiving commercial research funds from Daiichi Sankyo. YA is a consultant/advisory board member of Daiichi Sankyo, Kowa, GlaxoSmithKline, Novartis, PAREXEL International, and Boehringer Ingelheim, and reports receiving commercial research fund from Novartis. YOku and MS are full-time employees of Daiichi Sankyo. TS and YS are consultant/advisory board members of Daiichi Sankyo. YOha has a leadership role and owns stock of Statcom, is a consultant/advisory board member of Daiichi Sankyo, reports receiving commercial research funds from Astellas Pharma and Takeda Pharmaceutical, and has received travel accommodations from Yakult. No potential conflicts of interest were disclosed by the other authors.

\section{AUTHOR CONTRIBUTIONS}

WI had full access to the data used in this study, drafted the paper and had final responsibility for the decision to submit for publication. KU, KM, CT, YT, HM, SS, KF, TM, MN, YY, AT, and KS recruited patients and collected data. WI, MS, and SM conducted statistical analyses of the data. WI, SM, YA, YOku, MS, TS, YOha, and YS constructed the study conception and designed the study, contributed to analysis and interpretation of the data. All authors contributed to the drafting or revising of the manuscript, and approved the final version.

\section{REFERENCES}

Ando Y, Saka H, Ando M, Sawa T, Muro K, Ueoka H, Yokoyama A, Saitoh S, Shimokata K, Hasegawa Y (2000) Polymorphisms of UDPglucuronosyltransferase gene and irinotecan toxicity: A pharmacogenetic analysis. Cancer Res 60: 6921-6926.

Carlini LE, Meropol NJ, Bever J, Andria ML, Hill T, Gold P, Rogatko A, Wang H, Blanchard RL (2005) UGT1A7 and UGT1A9 polymorphisms predict response and toxicity in colorectal cancer patients treated with capecitabine/irinotecan. Clin Cancer Res 11: 1226-1236.

Chen YJ, Hu F, Li CY, Fang JM, Chu L, Zhang X, Xu Q (2014) The association of UGT1A1* 6 and UGT1A1*28 with irinotecan-induced neutropenia in Asians: a meta-analysis. Biomarkers 19: 56-62.

Dias MM, McKinnon RA, Sorich MJ (2012) Impact of the UGT1A1*28 allele on response to irinotecan: a systematic review and meta-analysis. Pharmacogenomics 13: 889-899.

Dias MM, Pignon J-P, Karapetis CS, Boige V, Glimelius B, Kweekel DM, Lara PN, Laurent-Puig P, Martinez-Balibrea E, Páez D, Punt CJA, Redman MW, Toffoli G, Wadelius M, McKinnon RA, Sorich MJ (2014) The effect of the UGT1A1*28 allele on survival after irinotecan-based chemotherapy: a collaborative meta-analysis. Pharmacogenomics J 14: $424-431$.

Goto A, Yamada Y, Yasui H, Kato K, Hamaguchi T, Muro K, Shimada Y, Shirao K (2006) Phase II study of combination therapy with S-1 and irinotecan in patients with advanced colorectal cancer. Ann Oncol 17: 968-973.

Greenland S (1995) Dose-response and trend analysis in epidemiology: Alternatives to categorical analysis. Epidemiology 6: 356-365.

Han JY, Lim HS, Shin ES, Yoo YK, Park YH, Lee JE, Jang IJ, Lee DH, Lee JS (2006) Comprehensive analysis of UGT1A polymorphisms predictive for pharmacokinetics and treatment outcome in patients with non-small-cell lung cancer treated with irinotecan and cisplatin. J Clin Oncol 24: 2237-2244.
Hazama S, Mishima H, Tsunedomi R, Okuyama Y, Kato T, Takahashi K, Nozawa H, Ando H, Kobayashi M, Takemoto H, Nagata N, Kanekiyo S, Inoue Y, Hamamoto Y, Fujita Y, Hinoda Y, Okayama N, Oba K, Sakamoto J, Oka M (2013) UGT1A1*6,1A7*3, and $1 A 9^{\star} 22$ genotypes predict severe neutropenia in FOLFIRI-treated metastatic colorectal cancer in two prospective studies in Japan. Cancer Sci 104: 1662-1669.

Hoskins JM, Goldberg RM, Qu P, Ibrahim JG, McLeod HL (2007) UGT1A1 28 genotype and irinotecan-induced neutropenia: Dose matters. J Natl Cancer Inst 99: 1290-1295.

Innocenti F, Undevia SD, Iyer L, Chen PX, Das S, Kocherginsky M, Karrison T, Janisch L, Ramírez J, Rudin CM, Vokes EE, Ratain MJ (2004) Genetic variants in the UDP-glucuronosyltranferase 1A1 gene predict the risk of severe neutropenia of irinotecan. J Clin Oncol 22: 1382-1388.

Innocenti F, Ratain MJ (2006) Pharmacogenetics of irinotecan: clinical perspectives on the utility of genotyping. Pharmacogenomics 7: 1211-1221.

Innocenti F, Kroetz DL, Schuetz E, Dolan ME, Ramírez J, Relling M, Chen P, Das S, Rosner GL, Ratain MJ (2009) Comprehensive pharmacogenetic analysis of irinotecan neutropenia and pharmacokinetics. J Clin Oncol 27: 2604-2614.

Innocenti F, Schilsky RL, Ramírez J, Janisch L, Undevia S, House LK, Das S, Wu K, Turcich M, Marsh R, Karrison T, Maitland ML, Salgia R, Ratain MJ (2014) Dose-finding and pharmacokinetic study to optimize the dosing of irinotecan according to the UGT1A1 genotype of patients with cancer. J Clin Oncol 32: 2328-2334.

Iyer L, King CD, Whitington PF, Green MD, Roy SK, Tephly TR, Coffman BL, Ratain MJ (1998) Genetic predisposition to the metabolism of irinotecan (CPT-11). Role of uridine diphosphate glucuronosyltransferase isoform $1 \mathrm{~A} 1$ in the glucuronidation of its active metabolite (SN-38) in human liver microsomes. J Clin Invest 101: 847-854.

Komatsu Y, Yuki S, Sogabe S, Fukushima H, Iwanaga I, Kudo M, Tateyama M, Meguro T, Uebayashi M, Saga A, Sakata Y, Asaka M (2011) Phase II study of combined treatment with irinotecan and S-1 (IRIS) in patients with inoperable or recurrent advanced colorectal cancer (HGCSG0302). Oncology 80: 70-75.

Kweekel D, Guchelaar HJ, Gelderblom H (2008) Clinical and pharmacogenetic factors associated with irinotecan toxicity. Cancer Treat Rev 34: 656-669.

Little RJA, Rubin DB (2002) Statistical Analysis with Missing Data (ed 2). Wiley Interscience: Hoboken, NJ.

Liu CY, Chen PM, Chiou TJ, Liu JH, Lin JK, Lin TC, Chen WS, Jiang JK, Wang HS, Wang WS (2008) UGT1A1 ${ }^{\star} 28$ polymorphism predicts irinotecan-induced severe toxicities without affecting treatment outcome and survival in patients with metastatic colorectal carcinoma. Cancer 112: 1932-1940.

Liu X, Cheng D, Kuang Q, Liu G, Xu W (2013) Association between UGT1A1 ${ }^{\star} 28$ polymorphisms and clinical outcomes of irinotecan-based chemotherapies in colorectal cancer: A meta-analysis in Caucasians. PLoS One 8: e58489.

Liu X, Cheng D, Kuang Q, Liu G, Xu W (2014) Association of UGT1A1²8 polymorphisms with irinotecan-induced toxicities in colorectal cancer: a meta-analysis in Caucasians. Pharmacogenomics J 14: 120-129.

Marcuello E, Altés A, Menoyo A, Del Rio E, Gómez-Pardo M, Baiget M (2004) UGT1A1 gene variations and irinotecan treatment in patients with metastatic colorectal cancer. Br J Cancer 91: 678-682.

Marcuello E, Páez D, Paré L, Salazar J, Sebio A, Del Rio E, Baiget M (2011) A genotype-directed phase I-IV dose-finding study of irinotecan in combination with fluorouracil/leucovorin as first-line treatment in advanced colorectal cancer. $\mathrm{Br} J$ Cancer 105: 53-57.

McLeod HL, Sargent DJ, Marsh S, Green EM, King CR, Fuchs CS, Ramanathan RK, Williamson SK, Findlay BP, Thibodeau SN, Grothey A, Morton RF, Goldberg RM (2010) Pharmacogenetic predictors of adverse events and response to chemotherapy in metastatic colorectal cancer: Results from North American Gastrointestinal Intergroup Trial N9741. J Clin Oncol 28: 3227-3233.

Minami H, Sai K, Saeki M, Saito Y, Ozawa S, Suzuki K, Kaniwa N, Sawada J, Hamaguchi T, Yamamoto N, Shirao K, Yamada Y, Ohmatsu H, Kubota K, Yoshida T, Ohtsu A, Saijo N (2007) Irinotecan pharmacokinetics/ pharmacodynamics and UGT1A genetic polymorphisms in Japanese: roles of UGT1A1*6 and ${ }^{*} 28$. Pharmacogenet Genomics 17: 497-504.

Muro K, Boku N, Shimada Y, Tsuji A, Sameshima S, Baba H, Satoh T, Denda T, Ina K, Nishina T, Yamaguchi K, Takiuchi H, Esaki T, Tokunaga S, Kuwano H, Komatsu Y, Watanabe M, Hyodo I, Morita S, Sugihara K (2010) Irinotecan plus S-1 (IRIS) versus fluorouracil and 
folinic acid plus irinotecan (FOLFIRI) as second-line chemotherapy for metastatic colorectal cancer: a randomised phase $2 / 3$ non-inferiority study (FIRIS study). Lancet Oncol 11: 853-860.

Palomaki GE, Bradley LA, Douglas MP, Kolor K, Dotson WD (2009) Can UGT1A1 genotyping reduce morbidity and mortality in patients with metastatic colorectal cancer treated with irinotecan? An evidence-based review. Genet Med 11: 21-34.

Rouits E, Boisdron-Celle M, Dumont A, Guérin O, Morel A, Gamelin E (2004) Relevance of different UGT1A1 polymorphisms in irinotecaninduced toxicity: A molecular and clinical study of 75 patients. Clin Cancer Res 10: 5151-5159.

Sai K, Saeki M, Saito Y, Ozawa S, Katori N, Jinno H, Hasegawa R, Kaniwa N, Sawada J, Komamura K, Ueno K, Kamakura S, Kitakaze M, Kitamura Y, Kamatani N, Minami H, Ohtsu A, Shirao K, Yoshida T, Saijo N (2004) UGT1A1 haplotypes associated with reduced glucuronidation and increased serum bilirubin in irinotecan-administered Japanese patients with cancer. Clin Pharmacol Ther 75: 501-515.

Sai K, Saito Y, Maekawa K, Kim SR, Kaniwa N, Nishimaki-Mogami T, Sawada J, Shirao K, Hamaguchi T, Yamamoto N, Kunitoh H, Ohe Y, Yamada Y, Tamura T, Yoshida T, Matsumura Y, Ohtsu A, Saijo N, Minami H (2010) Additive effects of drug transporter genetic polymorphisms on irinotecan pharmacokinetics/pharmacodynamics in Japanese cancer patients. Cancer Chemother Pharmacol 66: 95-105.

Saltz L (2013) Systemic therapy for metastatic colorectal cancer. J Natl Compr Canc Netw 11: 649-652.

Sato T, Ura T, Yamada Y, Yamazaki K, Tsujinaka T, Munakata M, Nishina T, Okamura S, Esaki T, Sasaki Y, Koizumi W, Kakeji Y, Ishizuka N, Hyodo I, Sakata Y (2011) Genotype-directed, dose-finding study of irinotecan in cancer patients with $U G T 1 A 1^{*} 28$ and/or $U G T 1 A 1^{*} 6$ polymorphisms. Cancer Sci 102: 1868-1873.

Senter PD, Beam KS, Mixan B, Wahl AF (2001) Identification and activities of human carboxylesterases for the activation of CPT-11, a clinically approved anticancer drug. Bioconjug Chem 12: 1074-1080.

Shimada Y, Yoshino M, Wakui A, Nakao I, Futatsuki K, Sakata Y, Kambe M, Taguchi T, Ogawa N. the CPT-11 Gastrointestinal Cancer Study Group (1993) Phase II study of CPT-11, a new camptothecin derivative, in metastatic colorectal cancer. J Clin Oncol 11: 909-913.

Shiozawa T, Tadokoro J, Fujiki T, Fujino K, Kakihata K, Masatani S, Morita S, Gemma A, Boku N (2013) Risk factors for severe adverse effects and treatment-related deaths in Japanese patients treated with irinotecanbased chemotherapy: A postmarketing survey. Jpn J Clin Oncol 43: 483-491.

Toffoli G, Cecchin E, Gasparini G, D’Andrea M, Azzarello G, Basso U, Mini E, Pessa S, De Mattia E, Lo Re G, Buonadonna A, Nobili S, De Paoli P, Innocenti F (2010) Genotype-driven phase I study of irinotecan administered in combination with fluorouracil/leucovorin in patients with metastatic colorectal cancer. J Clin Oncol 28: 866-871.

Xu G, Zhang W, Ma MK, McLeod HL (2002) Human carboxylesterase 2 is commonly expressed in tumor tissue and is correlated with activation of irinotecan. Clin Cancer Res 8: 2605-2611.

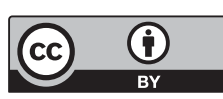

This work is licensed under the Creative Commons Attribution 4.0 International License. To view a copy of this license, visit http://creativecommons. org/licenses/by/4.0/

Supplementary Information accompanies this paper on British Journal of Cancer website (http://www.nature.com/bjc) 